\title{
Relación de calidad de vida y salud bucal de los adultos mayores en albergues de Tacna
}

\author{
Relationship between the quality of life and oral health of the elderly in shelters \\ in Tacna
}

\author{
Caroll Johana Uberlinda Lévano Villanueva ${ }^{\text {la }}$, Elva Alejandrina Maldonado de Zegarra ${ }^{2 b}$ \\ ${ }^{1}$ Investigador independiente. Tacna, Perú. \\ ${ }^{2}$ Escuela Profesional de Enfermeria, Universidad Nacional Jorge Basadre Grohmann. Tacna, Perú. \\ ${ }^{a}$ Maestro en Ciencias con Mención en Salud Pública. \\ ${ }^{\mathrm{b}}$ Docente, Doctora en Ciencias: Enfermería.
}

\section{Resumen}

Introducción: El siglo XXI se encuentra en una época de transición demográfica donde la población adulta mayor de 60 años está incrementándose. Por tal motivo, es importante prestar atención a una problemática de salud pública que podría desarrollarse en el futuro. En ese contexto, la salud bucal del adulto mayor es relevante en su calidad de vida, ya que las lesiones de caries podrían afectar su vida diaria debido al dolor, la imposibilidad de masticar, la dificultad para dormir entre otras afecciones. Por lo tanto, el odontólogo tendrá como nuevos desafíos la prevención y recuperación de la salud bucal, así como la promoción de un envejecimiento saludable. Objetivo: establecer la relación de calidad de vida y salud bucal de los adultos mayores de los albergues de la ciudad de Tacna en el año 2018. Materiales y Métodos: La muestra del estudio fue de 63 adultos mayores del hogar de ancianos “San José” y los adultos mayores del Centro Residencial Mixto del Adulto Mayor "San Pedro" de la Beneficencia Pública de Tacna; quienes fueron entrevistados para la variable calidad de vida mediante el índice de geriatría en salud oral (GOHAI). Tal instrumento evaluó la autopercepción de calidad de vida en relación a la salud bucal. Por otro lado, la variable salud bucal se analizó mediante el índice de piezas dentarias cariadas, perdidas y obturadas (CPOD). La hipótesis sugerida refirió la probabilidad que exista relación entre la calidad de vida y salud bucal. Para el análisis de los datos se utilizó el software SPSS 25 y se empleó la prueba estadística de Chi cuadrado con un nivel de significancia de $95 \%$. Resultados: Se identificó que el $77.8 \%$ de los adultos mayores consideraron baja su calidad de vida relacionada a la salud bucal. El índice de CPOD fue de 19.30 (DS 5.73), lo cual está en un nivel alto, con mayor porcentaje en piezas dentarias perdidas en un promedio de 13.03 (DS 8.89). Conclusión: No existe relación estadísticamente significativa; pero sí se encontró que al aumento del índice de CPOD disminuye la calidad de vida del adulto mayor en relación a la salud bucal.

Palabras clave: Adultos mayores, calidad de vida y salud bucal.

\section{Abstract}

Introduction: The 21 st century is in a time of demographic transition where the adult population over 60 is increasing. For this reason, it is important to pay attention to a public health problem that could develop in the future. In that context, the oral health of the elderly is relevant in their quality of life, since caries injuries could affect their daily lives due to pain, the inability to chew, difficulty sleeping among other conditions. Therefore, the dentist will have as new challenges the prevention and recovery of oral health, as well as the promotion of healthy aging. This research aims to establish the relationship of older adults' quality of life and oral health in shelters in Tacna city in 2018. Materials and Methods: The study sample was 63 older adults from the San Jose Nursing Home Care and San Pedro Mixed Residential Center of the Tacna Public Charity; who were interviewed for the variable quality of life through the oral health geriatrics index (GOHAI). This instrument evaluated the elderly's self-perception of their 
life quality in relation to their oral health. On the other hand, the oral health variable was analyzed using the CPOD index (decayed, lost and restored teeth index). The suggested hypothesis referred to the probability that there is a relationship between quality of life and oral health. For the data analysis, it was used the SPSS 25 software and the Chi-square statistical test with a level of significance of $95 \%$ was used. Results: It was identified that $77.8 \%$ of elderly people considered low their quality of life related to their oral health. The CPOD index was 19.30 (DS 5.73), which is at a high level, with a higher percentage of missing teeth by an average of 13.03 (DS 8.89). Conclusion: There is no statistically significant relationship; but it was found that the increase in the CPOD index decreases the quality of life of the elderly in relation to oral health.

Keywords: Elderly people, quality of life, oral health.

\section{Introducción}

La explosión demográfica del siglo XX abre paso, en el nuevo siglo, a la implosión geriátrica. Es, incluso, pertinente hablar de una suerte de globalización del envejecimiento, como consecuencia del avanzado proceso de transición demográfica e epidemiológica que se está viviendo (1).

La esperanza de vida en la población mundial se ha visto incrementada por un mayor cuidado en la nutrición, la salud y la optimización de las condiciones de vida, todo esto en combinación con los adelantos de las ciencias médicas. El reto de este siglo es afianzar una calidad de vida óptima para los adultos mayores y aplazar la aparición de discapacidades propias de la edad. Consecuentemente, los países se enfrentan a retos importantes para asegurar que sus sistemas sanitarios y sociales estén organizados para afrontar ese cambio demográfico(2).

Generalmente, esta población es la más desprotegida, ya que no cuenta con seguridad social, además de no ser económicamente activa. Su condición de salud propone un complicado esquema, el cual engloba enfermedades crónico-degenerativas, restricciones para la realización de actividades cotidianas, discapacidad y minusvalía. La morbilidad bucal se caracteriza por una elevada prevalencia de caries dental y periodontopatías, los que se constituyen en los principales factores responsables de la pérdida dental en este grupo de edad ocasionando trastornos físicos y psicosociales (3).

Considerando que las lesiones de caries desde el punto de vista del individuo que las adolece podrían llegar a impactar la calidad de vida debido al dolor, la imposibilidad de ejecutar acciones habituales como dormir, comer y hablar. Asimismo, problemas psicológicos y emocionales se encuentran relacionados, tales como irritabilidad, baja autoestima y apreciación negativa de cómo es percibido por otros adultos mayores. Por lo tanto, la caries es una enfermedad que no solo deteriora y afecta la salud bucal, sino también puede llegar a desestabilizar la calidad de vida del individuo y a quienes lo rodean $(4,5)$. De aquí se desprenden dos conceptos; el primero, la calidad de vida como el óptimo nivel de funcionamiento mental, físico y social, estado que incluye relaciones como la percepción de salud, la satisfacción y el bienestar (6). Ya el segundo, el concepto de calidad de vida relacionada a la salud oral, es decir, la valoración individual de cómo afectan los problemas orofaciales al bienestar. Estos problemas obedecen a factores disfuncionales, psicológicos, sociales y experiencia de dolor e incomodidad (7).

Las enfermedades bucales en su mayoría son irreversibles y las alteraciones tienden a ser mayores en los ancianos. En un corto plazo los odontólogos afrontarán el reto de brindar atención bucal a numerosos adultos mayores para conservar su cavidad bucal de manera funcional (8).

Existen diversos estudios sobre este tema, tales como el de Moya P, et.al. (9). En el referido estudio la edad media de los participantes fue de 71.33 años, quienes presentaban un índice COPD de 22.16. Este último fue aumentando con la edad. Se observó también que el $76.6 \%$ tuvo una percepción negativa de su calidad de vida. El autor concluye que los adultos mayores son un grupo de especial interés para la salud pública debido a la mala percepción de su calidad de vida y vulnerabilidad de su salud bucal. En sintonía, Zamora J. (10) mostró a través de su estudio que la autopercepción de la calidad de vida relacionada con la salud oral fue considerada como mala en un $34 \%$. En relación a las dimensiones planteadas en este estudio, la función psicosocial obtuvo un $36 \%$ de participantes que consideraron que no podían relacionarse con otras personas con comodidad. Este estudio determinó que de manera global la actividad diaria de los participantes se ve afectada por el estado de salud oral.

Los resultados de esta investigación apuntan a lograr nuevos conocimientos, los cuales sirvan como indicador para plantear estrategias de intervención y programar acciones para mejorar y rehabilitar la salud bucal de las personas que viven albergados en hogares geriátricos. Además, este estudio tiene relevancia social, ya que las enfermedades bucales son un importante problema de salud pública debido a su prevalencia y al impacto que tienen sobre los adultos mayores. La calidad de vida se ve afectada en diversos aspectos de la actividad cotidiana, tales como: las relaciones interpersonales, la función masticatoria y 
fono articular, la apariencia física e inclusive las oportunidades de trabajo. Es importante destacar que el objetivo de la investigación es determinar la relación entre calidad de vida y salud bucal del adulto mayor de los albergues de la ciudad de Tacna en el año 2018 con el propósito de contribuir a aumentar el conocimiento de una realidad que ya existe en el adulto mayor de nuestra localidad.

\section{Materiales y métodos}

Se realizó un estudio descriptivo, prospectivo, transversal (11), no experimental (12) y correlacional (13) en 63 adultos mayores de 60 años, siendo que 23 provinieron del Centro Residencial Mixto del Adulto Mayor "San Pedro" de la Beneficencia Pública de Tacna y 40 del Hogar de Ancianos "San José" en el año 2018. Se evaluó la salud bucal utilizando el índice de piezas dentarias cariadas, perdidas y obturadas (CPOD); por otro lado, se determinó la variable calidad de vida con el índice de geriatría en salud oral (GOHAI) a través de cuestionarios para cada participante del estudio a quien se le otorga puntuaciones sobre su salud bucal, contemplando la dimensión física, la dimensión psicosocial y la dimensión dolor e incomodidad.

Se tomó en cuenta los postulados de la Declaración de Helsinki de la Asociación Médica Mundial sobre las pautas éticas internacionales para la investigación biomédica en seres humanos (14). El presente estudio no implicó conflictos bioéticos debido a que se ejecutó con adultos mayores informados de los objetivos y de la metodología del estudio. Asimismo, se les solicitó firmar un consentimiento informado en el cual ratificaron su decisión de participar en el estudio por voluntad propia y que bajo ninguna circunstancia fueron obligados a hacer parte de la muestra.

Para el análisis de información se empleó estadística descriptiva y para la comparación de variables se utilizó la prueba estadística Chi cuadrado con un nivel de significancia de $95 \%$, considerando que un valor es significativo cuando $p<0.05$. Los resultados se presentaron en tablas descriptivas y comparativas, utilizando frecuencias absolutas y relativas. La información se procesó y analizó con el software estadístico SPSS Versión 25.

\section{Resultados}

Al examinar la Tabla 1, se observa que predomina el grupo etario de 80 a 90 años con 28 adultos mayores (44.4\%) y el género masculino con 39 adultos mayores.

\begin{tabular}{|c|c|c|c|c|c|c|}
\hline \multirow{3}{*}{ Edad } & \multicolumn{6}{|c|}{ Género } \\
\hline & \multicolumn{2}{|c|}{ Femenino } & \multicolumn{2}{|c|}{ Masculino } & \multicolumn{2}{|c|}{ Total } \\
\hline & n & $\%$ & n & $\%$ & n & $\%$ \\
\hline 60 a 69 & 2 & 8. 3 & 6 & 15. 3 & 8 & 12. 7 \\
\hline 70 a 79 & 9 & 37. 5 & 15 & 38. 5 & 24 & 38. 1 \\
\hline 80 a 89 & 10 & 41. 6 & 18 & 46. 2 & 28 & 44. 4 \\
\hline 90 a más & 3 & 12. 5 & 0 & 0.0 & 3 & 4. 8 \\
\hline Total & 24 & 100 & 39 & 100 & 63 & 100 \\
\hline
\end{tabular}

En la Tabla 2, la calidad de vida baja relacionada a la salud oral de los adultos mayores tuvo un $77.8 \%$ y la calidad de vida alta en salud oral tuvo un $22.2 \%$. El promedio del GOHAI fue de 42.84 puntos (Rango 12-60), lo cual indica que los examinados en su mayoría tenían una mala autopercepción de su salud bucal.

\begin{tabular}{|c|c|c|c|c|c|c|c|}
\hline \multirow[t]{2}{*}{$\begin{array}{l}\text { Calidad } \\
\text { de vida }\end{array}$} & \multicolumn{2}{|c|}{$\begin{array}{l}\text { Albergue } \\
\text { San José }\end{array}$} & \multicolumn{2}{|c|}{$\begin{array}{l}\text { Albergue } \\
\text { San Pedro }\end{array}$} & \multicolumn{2}{|c|}{ Total } & \multirow[b]{2}{*}{ Media } \\
\hline & n & $\%$ & n & $\%$ & n & $\%$ & \\
\hline Alta & 08 & 20 & 06 & 26. 1 & 14 & 22.2 & \\
\hline Baja & 32 & 80 & 17 & 73. 9 & 49 & 77.8 & \\
\hline Total & 40 & 100 & 23 & 100. 0 & 63 & 100.0 & 42.84 \\
\hline
\end{tabular}

Al analizar la Tabla 3, las dimensiones del índice de salud oral geriátrico (GOHAI), se encontró que la dimensión psicosocial fue el componente reportado con mayor promedio de 21.68 (DS 5.48), seguido de la dimensión física con un promedio de 14.67 (DS 4.12) y, por último, la dimensión dolor e incomodidad con un promedio de 6.49 (DS 1.94). 


\begin{tabular}{|c|c|c|c|c|}
\hline $\begin{array}{l}\text { Dimensiones } \\
\text { GOHAI }\end{array}$ & Albergues & $\mathbf{n}$ & Media & DS \\
\hline \multicolumn{5}{|l|}{ Dimensión } \\
\hline \multirow[t]{3}{*}{ Física } & $\begin{array}{l}\text { Albergue } \\
\text { San José }\end{array}$ & 40 & 14. 60 & 3.86 \\
\hline & $\begin{array}{l}\text { Albergue } \\
\text { San Pedro }\end{array}$ & 23 & 14. 78 & 4.62 \\
\hline & Total & 63 & 14.67 & 4.12 \\
\hline \multicolumn{5}{|l|}{ Dimensión } \\
\hline \multirow[t]{3}{*}{ Psicosocial } & $\begin{array}{l}\text { Albergue } \\
\text { San José }\end{array}$ & 40 & 21. 68 & 5.79 \\
\hline & $\begin{array}{l}\text { Albergue } \\
\text { San Pedro }\end{array}$ & 23 & 21. 70 & 5.01 \\
\hline & Total & 63 & 21.68 & 5.48 \\
\hline \multicolumn{5}{|l|}{ Dimensión } \\
\hline \multirow[t]{3}{*}{$\begin{array}{l}\text { Dolor e } \\
\text { incomodidad }\end{array}$} & $\begin{array}{l}\text { Albergue } \\
\text { San José }\end{array}$ & 40 & 6. 83 & 2.24 \\
\hline & $\begin{array}{l}\text { Albergue } \\
\text { San Pedro }\end{array}$ & 23 & 5. 91 & 1.08 \\
\hline & Total & 63 & 6.49 & 1.94 \\
\hline
\end{tabular}

La Tabla 4 muestra que el índice CPOD es de19.30, existiendo un promedio mayor en las piezas dentarias perdidas con 13.02 (DS 8.87). Esto indica que existe en los examinados mayor pérdida dentaria, lo cual es un hallazgo común en pacientes adultos mayores.

\section{Tabla 4. Estado de salud bucal (índice de CPOD) de los adultos mayores de los albergues de la ciudad de Tacna-2018}

\begin{tabular}{|c|c|c|c|c|c|c|}
\hline $\begin{array}{l}\text { Índice de } \\
\text { CPOD }\end{array}$ & Albergues & $\mathbf{n}$ & Media & DS & $\mathbf{T}$ & $\mathbf{P}$ \\
\hline \multicolumn{7}{|l|}{ Piezas } \\
\hline Dentarias & $\begin{array}{l}\text { Albergue } \\
\text { San José }\end{array}$ & 40 & 4.20 & 3.65 & & \\
\hline \multirow[t]{2}{*}{ Cariadas } & $\begin{array}{l}\text { Albergue } \\
\text { San José }\end{array}$ & 23 & 6.35 & 4. 34 & & \\
\hline & Total & 63 & 4.98 & 4. 02 & 2.10 & 0.040 \\
\hline \multicolumn{7}{|l|}{ Piezas } \\
\hline Dentarias & $\begin{array}{l}\text { Albergue } \\
\text { San José }\end{array}$ & 40 & 13.50 & 8.73 & & \\
\hline \multirow[t]{2}{*}{ Perdidas } & $\begin{array}{l}\text { Albergue } \\
\text { San Pedro }\end{array}$ & 23 & 12.17 & 9.24 & & \\
\hline & Total & 63 & 13.02 & 8.87 & 0.57 & 0.572 \\
\hline \multicolumn{7}{|l|}{ Piezas } \\
\hline Dentarias & $\begin{array}{l}\text { Albergue } \\
\text { San José }\end{array}$ & 40 & 1.50 & 1.77 & & \\
\hline \multirow[t]{2}{*}{ Obturadas } & $\begin{array}{l}\text { Albergue } \\
\text { San Pedro }\end{array}$ & 23 & 0.91 & 1. 38 & & \\
\hline & Total & 63 & 1.29 & 1.65 & 1.37 & 0.176 \\
\hline Total & & & 19.30 & & & \\
\hline
\end{tabular}

Los resultados mostrados en la Tabla 5 indican que la percepción de calidad de vida de salud oral en los adultos mayores es baja con 49 entrevistados. También, el índice CPOD se presenta con mayor frecuencia en el nivel alto con 37 examinados.

Tabla 5. Relación de calidad de vida y salud bucal
de los adultos mayores de los albergues de
la ciudad de Tacna-2018

\section{Discusión}

El estudio se realizó a 63 adultos mayores residentes en el Albergue San José (40) y Albergue San Pedro (23); siendo el grupo etario de 80 a más años de edad con mayor presencia de $44.4 \%$ (Tabla 1), datos que asemejan al estudio realizado por Oñate M. (15). En cuanto al género, predominó el masculino con 39 adultos mayores (Tabla 1), similar al estudio de Gallardo A. et al. (16); datos que contrastan con las investigaciones de Duque V. et al. (17) y Almirón P. et al. (18), en donde la mayoría de participantes fue del género femenino.

Los resultados del cuestionario de índice de salud oral geriátrico para evaluar calidad de vida (Tabla 2) son similares al estudio de Gallardo A, Picasso M, et al. (16) y Duran D, Moya P, et al. (19), en donde la calidad de vida resultó negativa-baja en un $77.4 \%$ utilizando el mismo instrumento de nuestra investigación. En cuanto a las dimensiones del cuestionario (GOHAI) (Tabla 3); la dimensión psicosocial resultó con un promedio de 21.68 , similar al estudio de Fuentes J, et al. (3) donde la dimensión psicosocial obtuvo mayor respuesta. Aquí, los participantes manifestaron sentimientos de inconformidad e insatisfacción con el estado de su boca debido a diferentes causas biológicas y socioculturales, lo que les impide desarrollarse de la mejor manera en su entorno social.

El índice CPOD resultó con un promedio de 19.30 y con un intervalo de 0 a 28 dientes (Tabla 4). Este resultado es una categoría menor a la encontrada en los estudios de Moya P. et al. (9), donde el CPOD es de 22.16 y Reyes J. et al. (20), quien encontró un índice CPOD considerado bajo (6.97). En cuanto al resultado de caries dental el mayor promedio es el de piezas dentarias perdidas con 13.04 (Tabla 4), lo que concuerda con el estudio de Roncal I. (21), donde la media de las piezas perdidas es de 11.29. Es importante mencionar que los resultados fueron similares por ser 
instituciones semejantes y, por tanto, con las mismas necesidades de atención dental.

En relación al grado del índice CPOD, este se presentó en un nivel alto con 37 entrevistados (Tabla 5); similar a los estudios de Sánchez M. (22) y Roncal I. (21), pero discordando con Moya P. et al. (8) y Reyes J. et al. (20). Estos últimos categorizan como grave el elevado índice de cariados, perdidos y obturados (CPOD), lo cual motiva a formular programas dirigidos a servicios dentales y educación para la salud. Estos programas pretenden promover el autocuidado de las piezas dentarias lo mismo desde una higiene oral adecuada como desde controles en la consulta dental.

Con respecto a los resultados obtenidos en la investigación según las pruebas estadísticas, no existe relación entre la calidad de vida y salud bucal de los adultos mayores de los albergues de la ciudad de Tacna en el año 2018 (Tabla 5). En concordancia con el estudio de Roncal I. (21); se comprobó que existían altos índices de CPOD y mala autopercepción de la salud bucal de manera individual; no obstante, en conjunto la relación entre ellas fue negativa.

Analizamos que aunque los adultos mayores tengan problemas o enfermedad bucal, ellos tienden a valorar positivamente su aspecto bucal, por ello, es difícil que informen valores negativos. Esas respuestas se contradicen y nos inducen a pensar en que el adulto mayor ya no tiene mucho interés en recuperar su salud oral y solo desea vivir de una manera digna donde pueda tener una buena interrelación personal y alimentarse de manera adecuada.

\section{Limitaciones del estudio}

El presente estudio presentó limitaciones, ya que resultó difícil que el adulto mayor respondiera por sí solo la encuesta y se decidió cambiar la técnica de encuesta a entrevista directa. Se comprobó que el adulto mayor regularmente no proporciona toda la información requerida debido a que cree que sus afecciones de salud forman parte del proceso de envejecimiento, además, sienten desconfianza y algunos no recordaban claramente datos lo que ocasionó un sesgo en la información.

\section{Conclusiones}

Se comprueba un mal estado de la salud bucal y una baja autopercepción de calidad de vida en salud oral. Según la prueba Chi cuadrado $x^{2}$, el $\mathrm{p}$ valor es = 0.37 mayor a 0.05 . Estos datos refieren que no existe significancia estadística; por lo tanto, en la contrastación de hipótesis se acepta la hipótesis nula y se determina que no existe relación directa entre la calidad de vida y la salud bucal de los adultos mayores de los albergues de la ciudad de Tacna en el 2018. Esto permite evidenciar la creciente necesidad que los profesionales de la salud afronten la conceptualización de calidad de vida relacionada con salud bucal, lo que motiva a detectar, controlar y realizar seguimiento de los problemas bucales como estrategia para menoscabar las alteraciones psicosociales y el mal estado de salud bucal de la población adulta mayor que vive en albergues y tienen derecho de atención como cualquier otra.

Se recomienda establecer alianzas estratégicas entre la Dirección Regional de Salud, las instituciones privadas y sociedad civil para desarrollar programas de atención del adulto mayor que se encuentra en albergues y no cuentan con atención de salud bucal; así como de actuar de manera oportuna en la rehabilitación de las condiciones físicas y subjetivas que puedan verse afectadas con el fin de lograr un envejecimiento digno y saludable. 


\section{Referencia bibliográficas}

1. Agar L. Transición demográfica y envejecimiento en américa latina y el caribe: hechos y reflexiones socio bioéticas. Acta bioeth 2001; [Sitio en internet]. 7(1): 27-41. [Citado 13 de noviembre de $\left.\begin{array}{llll}2 & 0 & 1 & 9\end{array}\right]$ D $\quad$ i s p o n i b l e e $n$ : https://scielo.conicyt.cl/scielo.php?script=sci artte xt\&pid=S1726-569X2001000100003\&lng=es

2. Organización Mundial de la Salud. Informe Mundial sobre el envejecimiento y la salud; 2015. [Sitio en internet]. Disponible en: https://www.who.int/ageing/publications/worldreport-2015/es/

3. Fuentes J, Sumano O, Sifuentes M, Zelocuatecalt A. Impacto de la salud bucal en la calidad de vida de los adultos mayores demandantes de atención dental. Revista Universitas Odontológica, 2010; 29, (63): 83-92. [Citado el 22 de setiembre del 2019]. Disponible en:

https://redalyc.org/pdf/2312/231216366010.pdf

4. Ayhan H, Suskean E, Yildirim S. The Effect of Nursing or rampant caries on height, body weight and hesd circumference. J Clin Pedistr Dent, 1996; 20 (3): 209-212.

5. Feitosa S, Colares V, Pinkham J. The psychosocial Effect of severe caries in 4 years old children in Recife, Pernambuco, Brazil. Cad Saude Pública, 2005; 21 (5): 1550.1556.

6. Bowling A. Measuring health. A review of quality of life Measurement Scales. $3^{\mathrm{a}}$, ed. Buckinham: Open Universit Press; 2005.

7. Inglehart MR, Bragamian RA. Oral health related quality of life: An introduction. In Oral Health related quality of life. Chicago: Quintenssence; 2002. pp. 1-6.

8. López G, López L, Nachon MG, Hernández D. Salud bucal, salud bucal percibida y calidad de vida en adultos mayores. Rev Med UV, 2014 [Citado 22 setiembre 2019]: 6-11. Disponible en:http://www.medigraphic.com/pdfs/veracruzana /muv-2014/muv142a.pdf

9. Moya P, Chappuzeau E, Caro JC, Monsalves MJ. Situación de salud oral y calidad de vida de los adultos mayores que asiste a centros de salud pública del área oriente de Santiago de Chile durante los años 2011-2012.[Sitio en internet] Revista Estomatológica Herediana. 2012 [Citado el 22 de setiembre de 2019]; 22(4):197-202. D i s p o n i b l e e $n$ : http://revistas.upch.edu.pe/index.php/REH/article/ view $/ 85$
10. Zamora J. Autopercepción de la calidad de vida relacionada con la salud oral en adultos mayores atendidos en el "Centro Integral del Adulto Mayor (CIAM)" del distrito de independencia en Lima Perú, 2016. Repos Inst - UCV [Sitio en Internet]. 2017 [Citado 22 de setiembre de 2019]; Disponible en: http://repositorio.ucv.edu.pe/handle/UCV/8868

11. Canales F, De Alvarado E y Pineda E. Metodología de la investigación manual de para el desarrollo de personal de salud.1994 [Sitio en internet] [Citado el 22 de setiembre del 2019] Cap. 6: 81. Disponible en: http://iris.paho.org/xmlui/handle/123456789/3132

12. Hernández Sampieri, R., Fernández, C., Baptista, P. Metodología de la investigación: Roberto Hernández Sampieri, Carlos Fernández Collado y Pilar Baptista Lucio. $6^{a}$. ed. México D.F.: McGrawHill; 2014. Cap.5 93-95. Disponible en: http://observatorio.epacartagena.gov.co/wpcontent/uploads/2017/08/metodologia-de-lainvestigacion-sexta-edicion.compressed.pdf

13. Cazau P. Introducción a la investigación en Ciencias Sociales. $3^{\mathrm{a}}$ ed. Buenos Aires; 2006 [Citado 22 de setiembre de 2019]. p.27. Disponible en: shorturl.at/chqzO

14. Manzini J. Declaración de Helsinki: principios éticos para la investigación médica sobre sujetos humanos. [Sitio en internet]. Acta Bioethica 2000; año VI, ${ }^{\circ}$ 2. [Citado 13 de noviembre de 2019]. Disponible en: https://scielo.conicyt.cl/pdf/abioe th/v6n2/art10.pdf

15. Oñate Torres M. Efecto de la salud oral en la calidad de vida de adultos mayores dependientes, comuna de Hualpén, Concepción-Chile 2015 [Internet] [Thesis]. Universidad Andrés Bello; 2015 [Citado 22 de setiembre del 2019]. Disponible en: http://repositorio.unab.cl/xmlui/handle/ria/2742

16. Gallardo A, Picasso MA, Huillca N, Ávalos JC. Calidad de vida relacionada con la salud oral en adultos mayores de una población peruana.[Sitio en internet] KIRU.2013; 10(2):145-50. [Citado el 22 de setiembre del 2019].Disponible en: www.usmp.edu.pe/odonto/servicio/2013/Kiruv.10. 2/ Kiru_v.10.2_Art.8.pdf.

17. Duque V, Tamayo J, Adudelo A, et al. Calidad de vida relacionada con la salud bucal de los adultos mayores que consultan a la IPS Universitaria de Medellín y sus factores asociados. Junio 2013. Revista CES odontología. 2013[Citado 22 de setiembre del 2019]; 26 (1). Disponible en: www. revistas.ces.edu.co. 
18. Almirón P, Denis E, Morales A, Britez E, Macchi A, Maidana $\mathrm{J}$, et al. Condiciones de salud oral en ancianos del club geriátrico "Abue-Club", Paraguay. Universidad del Pacífico Privada. Asunción, Paraguay. Mem. Inst. Investig. Cienc. Salud. 2016; 14(1):32-39.Disponible en http://scielo.iics.una.py/pdf/iics/v14n1/v14n1a06. pd

19. Durán D, Moya P, Aubert J, Becerra A, Lara-Lüer A, Monsalves MJ. Percepción de salud bucal en adultos mayores de dos comunas con desarrollos extremos en Chile. Rev. CES Odont 2016 [Citado 22 de setiembre 2019]; 29(1): 5-12. Disponible en: http://www.scielo.org.com

20 .Reyes J, et al. Determinación de la salud bucal en personas mayores institucionalizadas residentes en Lima, Perú. Repositorio Académico de la Universidad San Martín de Porres. Kiru. 2013
[Citado 22 de setiembre 2019]; 10(2):140-144. .Disponible en: www.repositorioacademico.usmp.edu.pe/handle/u smp/1928

21. Roncal I. Relación entre la autopercepción y el estado de salud bucal de los adultos mayores de los Centros Residenciales Gerontológicos de la Beneficencia Pública de Lima Metropolitana; 2013.Repositorio de la Universidad Peruana Cayetano Heredia. [Citado 22 de setiembre del 2019]. Disponible en: Repositorio .upch.edu.pe.

22. Sánchez M, Román M, Dávila R, GonzálezA. Salud bucal en pacientes adultos mayores y su asociación con la calidad de vida en la Clínica de Medicina Familiar de México. Revista de Especialidades Médico quirúrgicas, 2011; 16 (2): 110-115. [Citado 22 de setiembre del 2019]. Disponible en: https://www.redalyc.org/pdf/473/47319326010.pd $\mathrm{f}$

\section{Correspondencia:}

Caroll.levano67@gmail.com
Fecha de recepción : 09 de julio 2019

Fecha de aceptación : 07 de octubre de 2019 